\title{
AUTOCONCEPTO MULTIDIMENSIONAL Y ESTILOS DE VIDA SALUDABLE EN ESTUDIANTES DE ESTOMATOLOGIA - UNIVERSIDAD PRIVADA DE HUANCAYO FRANKLIN ROOSEVELT-2015
}

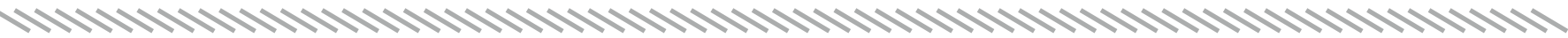 \\ MULTIDIMENSIONAL AUTOCONCEPT AND HEALTHY LIFESTYLES IN STOMATOLOGY STUDENTS "FRANKLIN ROOSEVELT" PRIVATE UNIVERSITY OF HUANCAYO - 2015}

\section{AUTORES: Mg. CD. Edgar Fernando Almonacid Sosa,' Mg. CD. Luis Alberto Cueva Buendía,}

Mg. PS. Evelyn Jeannet Jesús Balbín.

\section{RESUMEN}

OBJETIVOS: Conocer la relación entre los estilos de vida saludable y el autoconcepto multidimensional en estudiantes de Estomatología de la Universidad Privada de Huancayo Franklin Roosevelt - 2015.

MÉTODOS: Método general el científico y específicos el descriptivo y el estadístico. Tipo de investigación no experimental, diseño correlacional. La población fue de 158 estudiantes del I al VII ciclo excepto el ciclo IV donde no hubieron estudiantes; de ambos sexos, matriculados en sus respectivos ciclos, pertenecientes a la escuela profesional de Estomatología. La muestra fue 125 estudiantes seleccionados con muestreo probabilístico estratificado. La recolección de datos se realizó a través de la técnica; encuesta y escala de actitudes y los instrumentos fueron el cuestionario y la escala de actitudes tipo Likert. Los métodos que se utilizaron para el análisis y procesamiento de los datos fue a través del software SPSS V 20 y Excel 2010, los resultados se presentan en cuadros, tablas y gráficos.

RESULTADOS: Se evidenció que existe una relación significativa positiva alta entre estilos de vida saludable y autoconcepto multidimensional en los estudiantes de Estomatología de la Universidad Privada de Huancayo Franklin Roosevelt, sede Huancayo, durante el año 2015.

CONCLUSIÓN: Se ha determinado que existe una correlación significativa, positiva alta entre estilos de vida saludable y el autoconcepto dimensional satisfacción laboral en estudiantes de Estomatología de la Universidad Privada de Huancayo Franklin Roosevelt, sede Huancayo, durante el año 2015. Con un Rho de Spearman $=0,74$; nivel de significancia de 0,05 ; y la tc $(12,20)$ $>\mathrm{tt}(1,96)$.
PALABRA CLAVE: Estilos de vida saludable, autoconcepto multidimensional.

\section{ABSTRACT \\ OBJECTIVES: Know the relationship between healthy lifestyles and multidimensional self-concept in Stoma- tology students of Private University of Huancayo Franklin Roosevelt - 2015.}

METHODS: General scientific method and specifics the descriptive one and statistician. Type of non-experimental research, correlational design The population was 158 students from the 1 st to 7 th cycle except for cycle IV where there were no students; of both sexes, enrolled in their respective cycles, belonging to the professional school of Stomatology. The sample was 125 selected students with stratified probabilistic sampling. Data collection was done through the technique; survey and attitudes scale and instruments were the Likert-type questionnaire and attitude scale. The methods used for data analysis and processing were through SPSS V 20 software and Excel 2010, the results are presented in tables, charts and graphics.

RESULTS: It was evidenced that there is a significant positive relationship between healthy lifestyles and multidimensional self-concept in Stomatology students to Private University of Huancayo Franklin Roosevelt, Huancayo Headquarters, during the year 2015.

Conclusion: It has been determined that there is a significant, high positive correlation between healthy lifestyles and the dimensional self-concept of job satisfaction in Stomatology students to Franklin Roosevelt Private University of Huancayo, Huancayo Headquarters, during the year 2015. With a Spearman's Rho = 0.74 ; Significance level of 0,05 ; and the tc $(12,20)>t t$ $(1,96)$. 
KEY WORDS: Healthy lifestyles, multidimensional self-concept

\section{INTRODUCCIÓN}

Los primeros años de vida universitaria puede ser vista como un periodo de lucha por la independencia y madurez, como un tiempo de separación del control adulto. Por tanto, las conductas pueden adoptarse como formas de expresar un estatus parecido al de los adultos o una rebelión contra los valores adultos.

Además, es un periodo clave en el desarrollo del autoconcepto. Los cambios físicos, cognitivos y sociales que tienen lugar en esta etapa amenazan la estabilidad del autoconcepto. Se amplía el número de contextos en los que se desenvuelve el estudiante universitario; contextos en los que tiene que desempeñar diversos roles y que le permiten recibir una información más diversa acerca de sí mismo. Las conductas relacionadas con la salud pueden adoptarse como una forma de experimentar con distintas identidades, en las que dichas conductas pueden expresar o llevar a cabo un conjunto de atributos o características. $Y$ todo ligado desde luego con los estilos de vida, que pueden o no ser saludables, y que a su vez pueden favorecer en el rendimiento del estudiante universitario, siempre en cuando sea favorable.

Por ello este trabajo de investigación formuló la interrogante ¿Cuál es la relación entre el autoconcepto multidimensional y los estilos de vida saludable en estudiantes, se realizó una adecuada revisión teórica y empírica acerca de la relación entre el autoconcepto multidimensional y el estilo de vida relacionado con la salud, por consecuencia la justificación principal de este estudio fue teórica pues los resultados obtenidos permitieron conocer el grado de correlación o asociación que existe entre estas dos variables de interés.

Por otro lado el objetivo fue: determinar la relación que existe entre los estilos de vida saludable y el autoconcepto multidimensional. Y como hipótesis general: existe relación significativa entre los estilos de vida y el autoconcepto multidimensional en los estudiantes

Se obtuvo como resultado; que existe una relación significativa positiva alta entre estilos de vida saludable y autoconcepto multidimensional en los estudiantes de Estomatología de la Universidad Privada de Huancayo Franklin Roosevelt, sede Huancayo, durante el año 2015.

\section{MATERIAL Y MÉTODOS}

El método general utilizado fue el científico, que implica todas las etapas desde la descripción del problema, revisión bibliográfica, elaboración de objetivos, formulación de hipótesis y la aplicación de métodos específicos para hallar resultados, realizar la discusión y las conclusiones respectivas.
Además se emplearon métodos específicos como el analítico que distinguen los elementos del autoconcepto multidimensional y se procede a revisar ordenadamente cada uno de ellos por separado, lo mismo la variable estilos de vida; también se empleó el método correlacional para ver cómo se relacionan los estilos de vida saludable con autoconcepto. Se hizo uso del método lógico inductivo: Ya que en esta investigación partiendo de casos particulares (autoconcepto en estudiantes de una escuela académico profesional), buscaremos hacer generalizaciones.

\section{Además se utilizarán los siguientes métodos:}

- Para la variable: estudio de los estilos de vida saludable Se utilizó el inventario de conductas saludables, el instrumento traducido al castellano (Balaguer, 2002). Este instrumento fue diseñado por la oficina regional europea de la OMS, para el desarrollo de un programa de investigación internacional sobre los estilos de vida de los adolescentes, que fue adaptado por los autores de la presente investigación para aplicarse en estudiantes universitarios.

- Para la variable: estudio del autoconcepto multidimensional

El instrumento elegido para evaluar el autoconcepto y la autoestima es el Perfil de Autopercepciones para adolescentes SPPC (Harter, 1985). Este instrumento se construyó bajo el supuesto de la multidimensionalidad del autoconcepto y bajo la noción de que la autovalía global se puede evaluar directamente, así como independientemente de las autopercepciones relacionadas con dominios específicos. Concretamente, se halla teóricamente vinculado al modelo de los factores correlacionados descrito en el marco teórico. El SPPC está compuesto de 36 ítems que evalúan 5 dominios específicos del autoconcepto (comportamiento/conducta, aceptación social, competencia académica, competencia deportiva y apariencia física) y una escala independiente de autovalía global, en este caso este instrumento no se modificó en ninguno de sus componentes.

\section{RESULTADOS}

Se evidenció que existe una relación significativa positiva alta entre estilos de vida saludable y autoconcepto multidimensional en los estudiantes de Estomatología de la Universidad Privada de Huancayo Franklin Roosevelt, sede Huancayo, durante el año 2015.

\section{DISCUSIÓN}

En relación al objetivo general fue: conocer la relación entre los estilos de vida saludable y el autoconcepto multidimensional en estudiantes de Estomatología de la Universidad Privada de Huancayo Franklin Roosevelt, sede Huancayo, durante el año 2015. Se obtuvo una correlación positiva alta, conforme a los resultados de 
la Rho de Spearman, con 0,74. Y después de ser sometido a la prueba $T$, presentó un valor de 12,20, mayor que la t teórica de 1,96, al 0,05 de nivel de significancia. Comparando lo encontrado en la presente investigación con Moreno2 se puede decir que dicho autor encontró diferencias entre el autoconcepto de los varones y las mujeres en un grupo de adolescentes, es así que los varones tienen percepciones positivas en relación a los deportes y apariencia física mientras que las mujeres tienen mayores percepciones respecto a su comportamiento.

\section{CONCLUSIONES}

Se ha determinado que existe una correlación significativa, positiva alta entre estilos de vida saludable y el autoconcepto dimensional satisfacción laboral en estudiantes de Estomatología de la Universidad Privada de Huancayo Franklin Roosevelt, sede Huancayo, durante el año 2015. Con un Rho de Spearman =0,74; nivel de significancia de 0,05 ; y la tc $(12,20)>\mathrm{tt}(1,96)$.

- Se ha identificado que el porcentaje de los estudiantes de Estomatología de la Universidad Privada de Huancayo Franklin Roosevelt, sede Huancayo, durante el año 2015 que practican estilos de vida saludable es del $68,8 \%$.

- Se ha identificado que el porcentaje del tipo de autoconcepto de los estudiantes de Estomatología de la Universidad Privada de Huancayo Franklin Roosevelt, sede Huancayo, durante el año 2015 fue del 56,8\% para el autoconcepto positivo y el $43,2 \%$ para el autoconcepto negativo.

- Se ha identificado que la frecuencia y porcentaje de los estudiantes de Estomatología de la Universidad Privada de Huancayo Franklin Roosevelt, sede Huancayo, durante el año 2015 de acuerdo al sexo y edad. Fue el mayor porcentaje son estudiantes de sexo masculino con un $52 \%$ y en cuanto a la edad son más los estudiantes entre los 26 y 30 años, con un porcentaje del $36 \%$.

- Se ha determinado que existe una correlación significativa positiva alta entre los estilos de vida y la dimensión comportamiento del autoconcepto en estudiantes de Estomatología de la Universidad Privada de Huancayo Franklin Roosevelt, sede Huancayo, durante el año 2015. Con un Rho de Spearman $=0,729$ nivel de significancia de 0,05 ; y la tc $(11,81)>$ tt $(1,96)$.

- Se ha determinado que existe una correlación significativa positiva alta entre los estilos de vida y la dimensión aceptación social del autoconcepto en estudiantes de Estomatología de la Universidad Privada de Huancayo Franklin Roosevelt, sede Huancayo, durante el año 2015. Con un Rho de Spearman $=0,764$ nivel de significancia de 0,05 ; y la tc $(13,13)>$ tt $(1,96)$.

- Se ha determinado que existe una correlación significativa positiva alta entre los estilos de vida y la dimensión competencia académica del autoconcepto en estudiantes de Estomatología de la Universidad Privada de Huancayo Franklin Roosevelt, sede Huancayo, durante el año 2015. Con un Rho de Spearman = 0,718 nivel de significancia de 0,05 ; y la tc $(11,44)>t t(1,96)$.

- Se ha determinado que existe una correlación significativa positiva alta entre los estilos de vida y la dimensión apariencia física del autoconcepto en estudiantes de Estomatología de la Universidad Privada de Huancayo Franklin Roosevelt, sede Huancayo, durante el año 2015. Con un Rho de Spearman $=0,751$ nivel de significancia de 0,05 ; y la tc $(12,61)>$ tt $(1,96)$.

- Se ha determinado que existe una correlación significativa positiva alta entre los estilos de vida y la dimensión competencia deportiva del autoconcepto en estudiantes de Estomatología de la Universidad Privada de Huancayo Franklin Roosevelt, sede Huancayo, durante el año 2015. Con un Rho de Spearman $=0,797$ nivel de significancia de 0,05 ; y la tc $(14,63)>t t(1,96)$.

AGRADECIMIENTOS: A las autoridades administrativas de la Universidad Privada de Huancayo Franklin Roosevelt, por permitirnos la realización de la presente investigación. Y del mismo modo a los estudiantes que brindaron su colaboración en la aplicación de los instrumentos de investigación

\section{REFERENCIAS BIBLIOGRÁFICAS}

1. Elliot, D.S. (1993). Health-enhancing and health-compromising lifestyles. En S.G. Millstein, A.C. Petersen, y E.O. Nightingale (Ed.), Promoting the health of adolescents. New directions for the twenty-first century (pp. 119-145). Oxford, Oxford University Press.

2. Moreno Y. Un estudio de la influencia del autoconcepto multidimensional sobre el estilo de vida saludable en la adolescencia temprana [TESIS DOCTORAL] Valencia. Universidad de Valencia, Facultad de Psicología.

3. Moore, S., Laflin, M.T., \& Weis, D.L. (1996). The role of cultural norms in the self-esteem and drug use relationship. Adolescence, 31(123), 523-542.

4. Romero, E., Luengo, M.A., \& Otero-López, J.M. (1995). La relación entre autoestima y consumo de drogas en los adolescentes: un análisis longitudinal. Revista de Psicología Social, 10(2), 149-159.

5. Rosenberg, M. (1965). Society and the adolescent self-image. Princeton, NJ: Princeton University Press. 6. Pandina, R.J., \& Schuele, J.A. (1983). Psychosocial correlates of alcohol and drug use of adolescent students and adolescents in treatment. Journal of Studies on Alcohol, 44, 950-973.

7. Abernathy, T. J., Massad, L., \& Romano-Dwyer, L. (1995). The relationship between smoking and self-esteem. Adolescence, 30, 899-907.

8.Butler, J.T. (1982). Early adolescent alcohol consumption and self-concept, social class and knowledge of alcohol. Journal of Studies on Alcohol, 43(5), 603-607.

9. Coopersmith, S. (1959). A method for determining types of self-esteem. Journal of Abnormal and Social Psychology, 59, 87-94.

10. Mclnman, A.D., \& Grove, J.R. (1991). Multidimensional self-concept, cigarette smoking, 
and intentions to smoke in adolescents. Australian Psychologist, 26(3), 192-196.

11. Ansbacher, H.L. (1967). Lifestyle: A Historical and systematic review. Journal of Individual Psychology, 23, 191-212.

12. Coreil, J., Levin, J.S., \& Jaco, G. (1992). Estilo de vida. Un concepto emergente en las ciencias sociomédicas. Clínica y Salud, 3 (3), 221-231.

13. Badura, B. (1982). Estilos de vida y salud: La perspectiva socio-ecológica. En J.I. Ruiz Olabuenaga (Ed.), Estilos de vida e investigación social (pp. 219-235). Bilbao:

Mensajero.

14. Rodríguez Marín, J. (1992). Estilos de vida y salud. Clínica y salud, 3 (3), 233-237.

15. Rodríguez Marín, J., \& García, J.A. (1995). Estilo de vida y salud. En. J.M. Latorre (Ed.)

16. WHO (1986). Life styles and health. Social Science and Medicine, 22, 2, 117-124.

17. Aarø, L.E., Wold, B., \& Kannas, L. (1986). Health behaviour in schoolchildren: A WHO Cross national Survey. Health Promotion, 1, 17-33. Ciencias Psicosociales Aplicadas II (pp. 25-34). Madrid: síntesis.

18. Nutbeam, D., Aaro, L., \& Catford, J. (1989). Understanding children's health behaviour: The implication for health promotion for young people. Social Science \& Medicine,

29 (3), 317-325.

19. Abel, T. (1991). Measuring health lifestyles in a comparative analysis: Theoretical issues and empirical findings. Social Science and Medicine, 32 (8), 899-908. 20. Abel, T., Cockerham, C., Lüeschen, G., \& Kunz, G. (1989). Health lifestyles and selfdirection in employment among American men: A test of the spillover effect. Social Science and Medicine, 28 (12), 1269-1274.

21. Breslow, L. (1990). Lifestyle, fitness, and health. En C. Bouchard. Exercise, fitness and health: the consensus statement (pp. 155-163). Human Kinetic. Champaign.

20. Donovan, J.E., Jessor, R., \& Costa, F.M. (1993). Structure of health-enhancing behavior in adolescence: A latent-variable approach. Journal of Health and Social Behavior, 34, 346-362.

\section{Fecha de Recepción: 06/02/2016 Fecha de Revisión: 04/04/2016}

\section{Correo electronico: ealmonacid@uroosevelt.edu.pe}

